\title{
Reproductive activity in captive female cheetahs (Acinonyx jubatus) assessed by faecal steroids
}

\author{
J. L. Brown ${ }^{1}$, D. E. Wildt ${ }^{1}$, N. Wielebnowski ${ }^{2}$, K. L. Goodrowe ${ }^{3}$, \\ L. H. Graham ${ }^{1}$, S. Wells ${ }^{4}$ and J. G. Howard ${ }^{1}$ \\ ${ }^{1}$ Conservation and Research Center, National Zoological Park, Smithsonian Institution, Front Royal, \\ VA 22630, USA; ${ }^{2}$ Department of Wildlife and Fisheries Biology, University of California, Davis, \\ CA 95616, USA; ${ }^{3}$ Metro Toronto Zoo, Scarborough, M1B 5K7, Ontario, Canada; and ${ }^{4}$ Department of \\ Mammals, National Zoological Park, Smithsonian Institution, Washington, DC 20008, USA
}

Faecal oestradiol and progestogen metabolite excretion was monitored in adult, female cheetahs (Acinonyx jubatus) $(n=26)$ for $1-24$ months. Increased faecal oestradiol excretion was associated with mating or equine chorionic gonadotrophin (eCG) administration for artificial insemination, whereas increased progestogen metabolites were observed during natural and human chorionic gonadotrophin (hCG)-induced pregnant and nonpregnant luteal phases. On the basis of oestradiol excretory patterns, duration of the oestrous cycle (mean \pm SEM) was $13.6 \pm 1.2$ days with high oestradiol concentrations lasting for $4.1 \pm 0.8$ days. In non-gonadotrophin-treated cheetahs, $75 \%$ showed evidence of oestrous cyclicity; however, none evaluated for 1 year or longer were continuously cyclic. Rather, cyclicity was interrupted by periods of anoestrus, often exceeding several months in duration. These inactive ovarian periods were unrelated to season and were not synchronous among females. Mean duration of gestation (breeding to parturition) was $94.2 \pm 0.5$ days, whereas duration of faecal progestogen metabolite excretion during the nonpregnant luteal phase was $51.2 \pm 3.5$ days. On the basis of progestogen metabolite evaluations, spontaneous ovulation (non-mating induced) occurred only once in two females (2 of 184 oestrous cycles; $1.1 \%$ ). Peak eCG-stimulated, preovulatory oestradiol concentrations were similar to those associated with natural oestrus, whereas progestogen metabolite profiles after hCG resembled those during pregnant and nonpregnant luteal phases after natural mating. In summary, results confirm that the cheetah is polyoestrus and ovulation is almost always induced. However, new evidence suggests that many females inexplicably experience periods of anoestrus unrelated to season, while $25 \%$ of the cheetahs examined expressed no ovarian activity during the study period.

\section{Introduction}

It is estimated that fewer than 15000 wild cheetahs (Acinonyx jubatus) remain in southern and eastern Africa, and their continued existence is threatened by many factors, including predation and competition by other carnivores, especially lions and hyenas, and extermination by humans (Laurenson et al., 1992; Marker-Kraus and Grisham, 1993; Caro, 1994). Cheetahs in captivity and in the wild also suffer from a lack of genetic diversity which may negatively impact reproductive function and affect long-term survival (O'Brien $e t$ al., 1983, 1985). Even so, the reproductive rate of free-ranging cheetahs appears to be relatively high with perhaps $80 \%$ of adults producing offspring during their lifetime (Laurenson et al., 1992). In contrast, the species has proven difficult to breed in captivity despite considerable effort (Guggisburg, 1975). Only about one-third

Received 1 September 1995 of zoo-maintained cheetahs have ever reproduced and infant mortality, usually related to maternal neglect, averages 30-40\% (Marker and O'Brien, 1989; Marker-Kraus and Grisham, 1993).

Possible causes of poor fertility in captivity were determined by conducting a reproductive survey of North American cheetahs (sanctioned by the Cheetah Species Survival Plan) between January 1990 and June 1991 (Wildt et al., 1993). In general, reproductive tract anatomy and pituitary function were normal in most adult females irrespective of breeding success. Furthermore, although male cheetahs naturally produce a high proportion of malformed spermatozoa (Wildt et al., 1983,1987 ), there were no differences in seminal quality between proven and unproven breeders (Wildt $e t$ al., 1993). In contrast, $>50 \%$ of females appeared acyclic on the basis of laparoscopic observations of inactive ovaries combined with parallel, one-time measurements of baseline circulating ovarian steroids. This survey was the first organized and 
comprehensive attempt ( 60 males: 68 females at 18 institutions) to identify possible biological causes of poor reproduction in cheetahs and suggested that poor fecundity in captivity may reflect suboptimal husbandry and management conditions rather than a fundamental loss in reproductive fitness. Longitudinal studies now are needed to evaluate more fully the dynamics of reproductive steroid secretion in this species.

In the present study, non-invasive faecal steroid monitoring was used to evaluate reproductive events in cheetahs to confirm and explain the apparent lack of ovarian cyclicity. In addition, new data were generated on the 'normality' of ovarian responses to exogenous hormonal ovulation induction and artificial insemination protocols by comparing faecal steroid profiles in pregnant versus nonpregnant animals after natural mating.

\section{Materials and Methods}

\section{Animals and faecal sample collection}

Study animals included adult, female cheetahs maintained at: the Phoenix Zoo, Phoenix, AZ $(n=4 ; 5.8 \pm 3.4$ years of age, range $=3-10$ years $)$; the Metro Toronto Zoo, Toronto $(n=3$; $5.7 \pm 1.7$ years of age, range $=3.5-9$ years); the White Oak Conservation Center, Yulee, FL $(n=8 ; 6.4 \pm 1.2$ years of age, range $=2.5-12$ years); the Sacramento Zoo, Sacramento, CA ( $n=2$; both 2.5 years of age); and Wildlife Safari, Winston, OR ( $n=6 ; 5.5 \pm 1.7$ years of age, range $=3-13$ years). Five animals at the White Oak Conservation Center and the two Sacramento Zoo cheetahs were monitored on two separate occasions. Three additional females maintained at the Caldwell Zoo, Tyler, TX $(8.3 \pm 2.2$ years of age, range $=4-11$ years $)$ were subjected to ovulation induction for laparoscopic artificial insemination (see below) twice, at an interval of eight months. Faecal samples were collected 3-7 times a week from all cheetahs for periods of 1-24 months and were stored frozen $\left(-20^{\circ} \mathrm{C}\right)$ in $50 \mathrm{ml}$ conical polypropylene vials until processed.

Cheetah management differed markedly among institutions making it impossible to correlate husbandry practices with specific biological events. Social groups and caging situations also varied throughout the year even within institutions. However, there was consistency in that all animals were exposed to natural fluctuations in photoperiod and each institution had a least one male housed within olfactory proximity to females. In general, females were housed with other females (at least occasionally) and, with the exception of one cheetah at the Wildlife Safari and two at the White Oak Conservation Center, all had been exposed to males for breeding (although not necessarily during the study period). Breeding strategies varied: some females were introduced to a male on a single day when she appeared in oestrus (affective behaviour, rolling, calling or lordodic posturing), whereas others were housed with a male for various time periods (days or weeks). Cheetahs at the Sacramento Zoo, Phoenix Zoo and White Oak Conservation Center were fed Nebraska Canine Diet (North Platte, NE), supplemented weekly with bones, chicken carcasses or horse ribs. Cheetahs at the Wildlife Safari were fed carcass meat only (horse, cow, deer, chicken, turkey) supplemented with calcium and vitamins. Animals at the Metro
Toronto Zoo were fed ground horsemeat supplemented with minerals/vitamins and whole carcasses (rabbit, guinea pig).

\section{Semen collection, induction of ovulation and artificial insemination}

Electroejaculates for artificial insemination were collected from two males at the Caldwell Zoo ( 6 and 7 years of age). Semen was collected under ketamine $\mathrm{HCl}$ (15-20 $\mathrm{mg} \mathrm{kg}^{-1}$, i.m.; Vetalar ${ }^{\prime \prime}$; Parke-Davis, Morris Plains, NJ) anaesthesia administered via a projectile dart as described by Howard et al. (1992). In brief, an AC sine-wave electroejaculator with rectal probe was used in a regimented sequence consisting of 80 incremental electrical stimuli (3-7 volts) given in an on-off pattern in three series over about $20 \mathrm{~min}$ (Wildt et al., 1983, 1987, 1993). Total ejaculate volume, sperm cell concentration and progressive motility of spermatozoa were determined as described by Wildt et al. (1983, 1987, 1993), and Howard et al. (1992). Each ejaculate was diluted (1:1) with Ham's F10 medium (Irvine Scientific, Santa Ana, CA) containing 5\% (v/v) heat-inactivated fetal calf serum (Irvine Scientific), centrifuged (at $300 \mathrm{~g}$ for $10 \mathrm{~min}$ ); the supernatant was discarded and the sperm pellet resuspended gently in $250-300 \mu \mathrm{l}$ of fresh Ham's F10 medium (Howard et al., 1992).

Cheetahs designated for artificial insemination were induced to ovulate using a gonadotrophin regimen established by Howard et al. (1992). In brief, equine chorionic gonadotrophin (eCG; 200 iu; Sigma Chemical Co., St Louis, MO) and human chorionic gonadotrophin (hCG; $100 \mathrm{iu}$; Sigma Chemical Co.) were injected i.m., $80 \mathrm{~h}$ apart to stimulate follicular development and ovulation, respectively. Intrauterine insemination was performed laparoscopically about $45 \mathrm{~h}$ after $\mathrm{hCG}$ injection using a method similar to that described by Howard et al. (1992). Anaesthesia was induced with ketamine $\mathrm{HCl}$ (5-10 mg $\mathrm{kg}^{-1}$, i.m.) and xylazine $\left(0.5-2 \mathrm{mg} \mathrm{kg}^{-1}\right.$, i.m; Rompun ${ }^{(\mathbf{R})}$, Miles Laboratory, Inc., Shawnee Mission, KS) administered via a projectile dart. Surgical anaesthesia was maintained with isoflurane gas-oxygen administered via intubation. Each cheetah was placed in a supine head-down position, a pneumoperitoneum produced and a $10 \mathrm{~mm}$ laparoscope (Olympus Corporation, Lake Success, NJ) inserted at the midline. An accessory grasping forcep was used to stabilize the uterine horn and an 18-gauge catheter (Sovereign ${ }^{(1)}$, Sherwood Medical, St Louis, MO) was inserted transabdominally into each uterine horn as a conduit for sterile polyethylene tubing (PE-10; Intramedic ${ }^{\mathrm{R}}$, Clay Adams Parsippany, NJ) containing about $10 \times 10^{6}$ motile spermatozoa in Ham's F10 medium. The PE tubing was placed into the uterine lumen beyond the tip of the catheter and the diluted spermatozoa (125-150 $\mu$ l per horn) were expelled.

\section{Faecal steroid analysis}

Faecal oestradiol and progestogen metabolites were extracted from samples as described by Brown et al. (1994, 1995). Briefly, samples were lyophilized, pulverized and about $0.2 \mathrm{~g}$ well-mixed powder boiled in $5 \mathrm{ml}$ aqueous ethanol $90 \%(\mathrm{v} / \mathrm{v})$ for $20 \mathrm{~min}$. After centrifuging at $500 \mathrm{~g}$ for $10 \mathrm{~min}$, supernatant was recovered and the pellet resuspended in $5 \mathrm{ml}$ $90 \%$ ethanol, vortexed for $1 \mathrm{~min}$ and re-centrifuged. Both 
ethanol supernatants were combined, dried completely and then redissolved in $1 \mathrm{ml}$ methanol. Extractants were vortexed ( $1 \mathrm{~min}$ ), placed in an ultrasonic cleaner for $30 \mathrm{~s}$ and re-vortexed (15 s). Samples were diluted (1:40 for oestradiol; 1:800I:80 000 for progestogens) in PBS $\left(0.01 \mathrm{~mol} \mathrm{PO}_{4} \mathrm{l}^{-1}, 0.14 \mathrm{~mol}\right.$ $\mathrm{NaCl} 1^{-1}, 0.5 \%(w / v)$ BSA, $\left.0.01 \%(w / v) \mathrm{NaN}_{3}\right)$ before analysis. Recovery of $\left[{ }^{3} \mathrm{H}\right]$ oestradiol and $\left[{ }^{14} \mathrm{C}\right]$ progesterone (New England Nuclear, Wilmington, DE) added to faecal samples before extraction exceeded $90 \%$.

Faecal oestradiol and progestogen metabolites were quantified using radioimmunoassays validated for cheetahs as described by Brown et al. (1994). The oestradiol radioimmunoassay relied upon an antibody provided by S. Wasser (Center for Wildlife Conservation, Seattle, WA) (Risler et al., 1987), a ${ }^{3} \mathrm{H}$-labelled oestradiol tracer (New England Nuclear) and oestradiol standards. This assay specifically quantified faecal oestradiol, with minimal crossreactivity $(\leq 2 \%)$ with other faecal oestrogen metabolites (oestradiol sulfate and oestrone). The progesterone radioimmunoassay relied upon a monoclonal progesterone antibody produced against 4-pregnen-11-ol-3,20-dione hemisuccinate:BSA (331; provided by J. Roser, University of California, Davis, CA), an ${ }^{125}$ I-labelled progesterone tracer (ICN Biomedical, Inc., Costa Mesa, CA) and progesterone standards. The assay specifically quantified the major conjugated progestogen metabolite(s) and several free pregnanolone epimers (Brown et al., 1994). Assay sensitivities, based on $90 \%$ of maximum binding, were 5 pg per tube and $7.5 \mathrm{pg}$ per tube for the oestradiol and progesterone assays, respectively. Intra- and interassay coefficients of variation were $<10 \%$ for both assays. All faecal data are expressed as $\mathrm{g}^{-1}$ dry mass.

\section{Statistical analyses}

Significant increases in faecal oestradiol concentrations were determined by an iterative process in which high values were excluded if they exceeded the mean $+1.5 \mathrm{sD}$. Baseline values were those remaining after all high values had been excluded. The duration of the oestrous cycle was calculated as the number of days between peaks in oestradiol concentrations (presumed to be associated with oestrus) for periods not exceeding 30 days (that is, > twice the estimated oestrous cycle duration: Eaton and Craig, 1973; Bertschinger et al., 1984; Asa et al., 1992). Interoestradiol peak intervals $>30$ days were considered as anoestrous periods. The number of days on which oestradiol was raised above baseline (indicative of oestrus) was calculated only during periods when faecal samples were collected for a minimum of five times per week. Data from females monitored $<60$ days and during pregnant and nonpregnant luteal phases were not included in oestrous cycle calculations. In females subjected to induction of ovulation and artificial insemination, baseline oestradiol concentrations were calculated from all samples before induction of ovulation. The beginning of the oestradiol surge was determined by a value that exceeded preceding values by $50 \%$. Basal progestogen metabolite concentrations were calculated from values preceding preovulatory oestradiol surges. Postovulatory increases in progestogen metabolite excretion were considered significant if values exceeded the mean \pm 2 SD of the preceding values and remained high for at least 1 week. Mean progestogen metabolite concentrations during pregnant and nonpregnant luteal phases contained values from the time of observed mating or artificial insemination to parturition or the sustained return of progestogen metabolite excretion to baseline values. Weekly or three times weekly means were calculated for each individual female and then averaged to provide the respective group means. Differences in preovulatory peak oestradiol concentrations or mean progestogen metabolite concentrations between pregnant and nonpregnant luteal phases, or gonadotrophin-treated versus naturally mated females, were determined using Student's + tests. Data are presented as means \pm SEM.

\section{Results}

\section{General observations}

On the basis of 184 cycles from 18 individuals, oestrous cycle duration was $13.6 \pm 1.2$ days (range, 5-30 days) with increases in oestradiol concentrations lasting $4.1 \pm 0.8$ days (range, $1-14$ days; $n=132$ cycles). When partitioned by duration, the percentages of oestrous cycles $\leq 7,8-13,14-19$ and $\geq 20$ days in length were $20,28,35$ and $17 \%$, respectively. There was considerable variation in the duration of the oestrous cycle, both within and among individuals. For females evaluated for $\geq 1$ year, overall mean duration of the oestrous cycle (average of all oestrous cycles for each individual) ranged from $10.4 \pm 1.0$ to $19.0 \pm 2.2$ days. Even within a female, oestrous cycle duration typically spanned the entire range from $<7$ to $>20$ days.

Baseline oestradiol concentrations generally ranged from 25-60 $\mathrm{ng} \mathrm{g}^{-\mathrm{I}}$ dry faecal mass with peak concentrations ranging from 100 to $750 \mathrm{ng} \mathrm{g}^{-1}$. There were no differences $(P>0.05)$ in peak preovulatory oestradiol concentrations between animals that conceived $\left(284.3 \pm 45.5 \mathrm{ng} \mathrm{g}^{-1}\right.$; $n=5)$ and those that mated but did not conceive (314.8 $\pm 41.9 \mathrm{ng} \mathrm{g}^{-1} ; n=8$ ) (Fig. 1). Similarly, there were no differences $(P>0.05)$ in preovulatory oestradiol concentrations between naturally-mated and eCG-treated (281.0 \pm $39.6 \mathrm{ng} \mathrm{g}^{-1} ; n=6$ ) females (Fig. 1). Peak oestradiol concentrations in nonmated females averaged $302.1 \pm 12.3 \mathrm{ng} \mathrm{g}^{-1}$ Faecal oestradiol excretion during pregnancy tended to remain at baseline values until several weeks before parturition, when concentrations increased up to tenfold and then declined after parturition (Fig. 1). In contrast, mean oestradiol concentrations during the nonpregnant luteal phase generally remained at baseline values, although random peaks occasionally occurred.

Average baseline faecal progestogen metabolite concentrations among individuals ranged from 0.7 to $6.0 \mu \mathrm{g} \mathrm{g}^{-1}$. Faecal progestogens in ovulating females increased within 1-10 days of the oestradiol surge. In pregnant females, concentrations remained 100 - to 400 -fold greater than baseline throughout gestation, rarely decreasing to less than 20-fold over baseline until near parturition (Fig. 1). There were no differences $(P>0.05)$ in overall mean progestogen metabolite concentrations between pregnant (202.9 $\left.\pm 15.3 \mu \mathrm{g} \mathrm{g}^{-1}\right)$ and nonpregnant $\left(240.6 \pm 26.4 \mu \mathrm{g} \mathrm{g}^{-1}\right)$ cheetahs during the period 

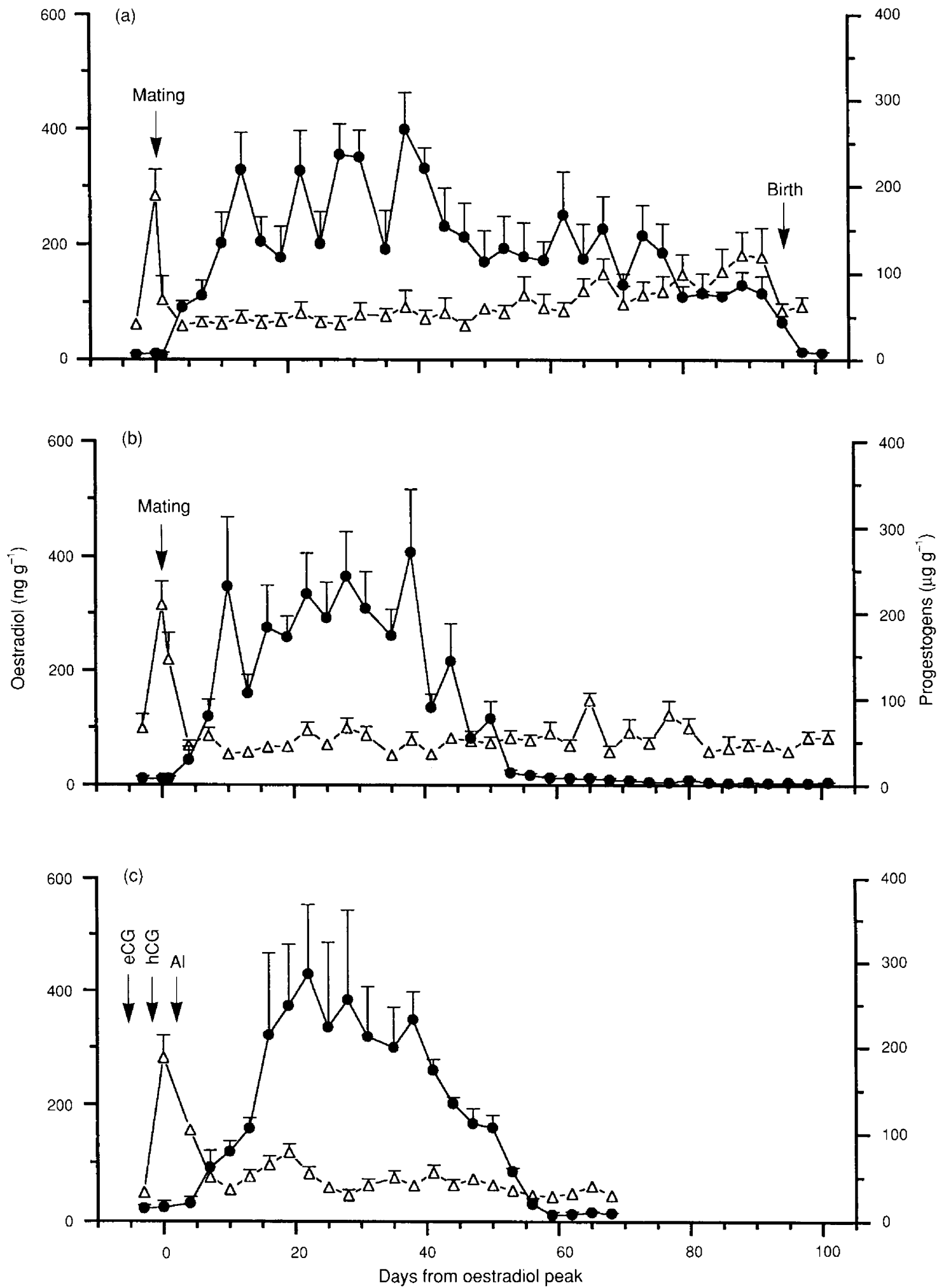

Fig. 1. Mean $( \pm$ SEM) faecal oestradiol $(\triangle)$ and progestogen $(\bullet)$ metabolite concentrations in (a) pregnant $(n=5)$ and $(b)$ nonpregnant $(n=8)$ cheetahs after natural mating and in (c) nonpregnant cheetahs after induction of ovulation by gonadotrophin and artificial insemination (AI) $(n=6)$. Data are aligned to the oestradiol peak (day 0 ).

of increased excretion or between the nonpregnant luteal phases of gonadotrophin-stimulated $\left(247.1 \pm 29.9 \mathrm{\mu g} \mathrm{g}^{-1}\right)$ versus naturally-mated individuals (Fig. 1). Mean duration of gestation (from the day of observed mating or preovulatory oestradiol surge to birth) was $94.2 \pm 0.5$ days (range, 93-96 days), whereas the duration of the nonpregnant luteal phase 

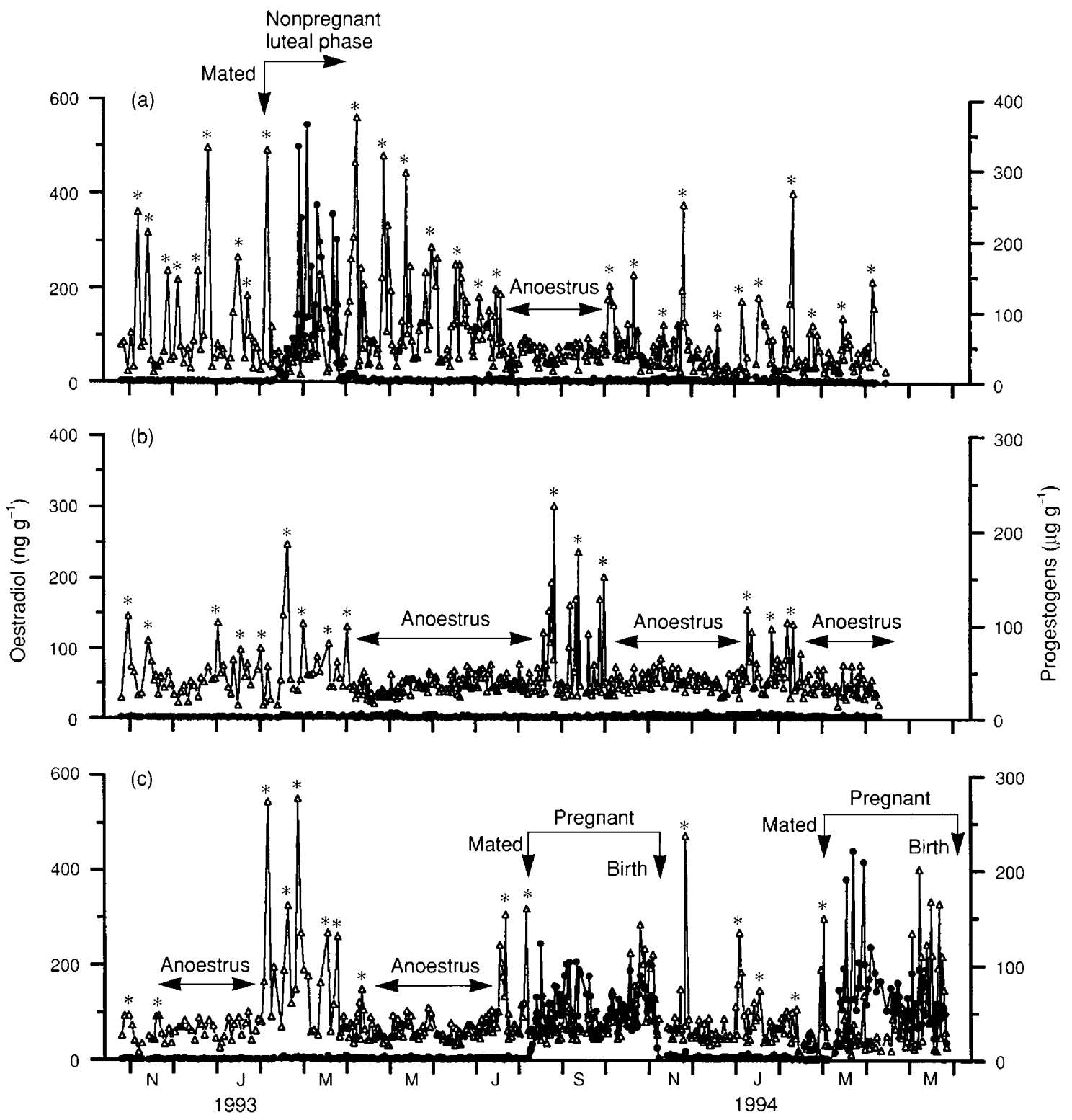

Fig. 2. Representative individual longitudinal profiles of faecal oestradiol $(\Delta)$ and progestogen ( $)$ metabolite concentrations in femaie cheetah at the Phoenix Zoo. Asterisks denote peaks in oestradiol excretion significantly above the baseline.

was about half $(P<0.05)$ that of pregnancy $(51.2 \pm 3.5$ days; range, 38-59 days).

\section{Longitudinal endocrine evaluations}

Eighteen of 24 cheetahs $(75 \%)$ monitored for 60 days or more exhibited some evidence of oestrous cyclicity on the basis of regular fluctuations in oestradiol excretion. In addition, ail individuals monitored $\geq 1$ year $(n=7)$ expressed cyclic activity, although none were continuously cyclic (Figs 2 and 3). Instead, follicular activity was interrupted by anoestrous periods, 2-5 months in duration, that were neither synchronous among females within facilities nor associated with season or other obvious environmental fac- tors. In females identified as acyclic, faecal monitoring had been conducted for 90 days or fewer. Two cheetahs (depicted in Fig. 2b,c) tended to express cyclic activity when the third female (Fig. 2a) was reproductively inactive (during a nonpregnant luteal phase or anoestrus). The cheetah in Fig. $2 a$ was older (10 years) and was cyclic about $80 \%$ of the time compared with the two younger sibling females (3 years; Fig. $2 b, c)$, each of which was cyclic about $40 \%$ of the time. Similarly, the cheetahs depicted in Fig. 3 also displayed periods of anoestrus that were not synchronous. In the female depicted in Fig. 2c, two pregnancies occurred within one year. At the end of the first pregnancy, a single cub was born and removed for hand-rearing which resulted in a resumption of ovarian cyclicity within one week. 


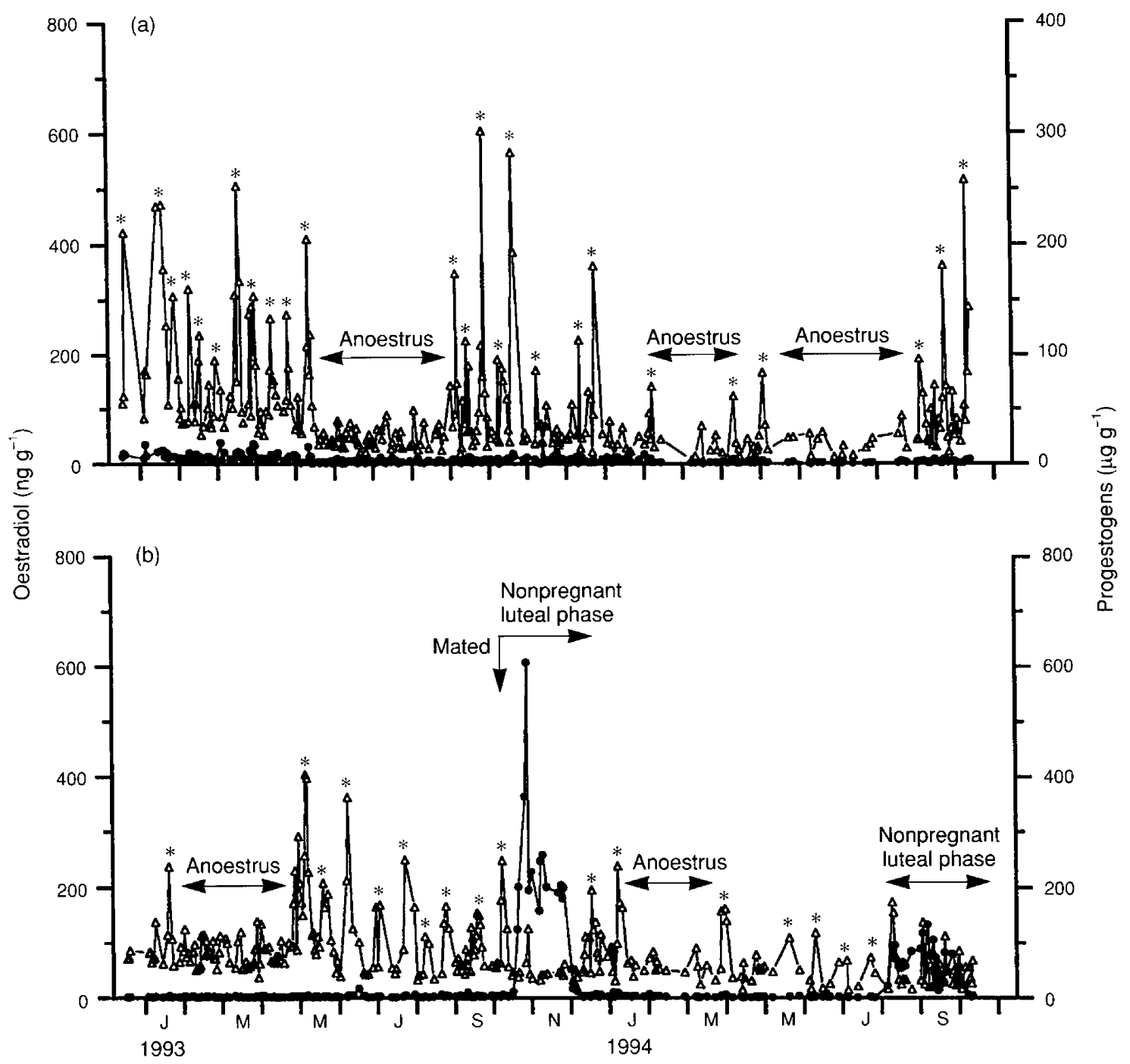

Fig. 3. Representative individual longitudinal profiles of faecal oestradiol $(\Delta)$ and progestogen $(\boldsymbol{O})$ metabolite concentrations in female cheetah at the Metro Toronto Zoo. Asterisks denote peaks in oestradiol excretion significantly above the baseline.

In almost all cases, episodic increases in oestradiol excretion (presumed indicative of oestrus) occurred without a subsequent rise in progestogen metabolite excretion in nonmated females (even those housed with other females), indicating a lack of spontaneous ovulation. However, two females were exceptions, exhibiting significant increases in faecal progestogen metabolite concentrations after an oestradiol surge in the absence of physical contact with a male. Progestogen excretory patterns in these two females were similar to those observed after induced ovulations, although overall concentrations tended to be lower (Fig. 4). In one case, increased progestogen excretion was observed within days after the female was relocated to a new enclosure and a male was moved into the adjacent pen on the same day (Fig. $4 a)$. In the other case, the female was translocated and a male introduced into her enclosure one week later. The male showed interest in the female (calling, approaches to female), but was removed from the enclosure before mounting occurred (Fig. 4b).
Endocrine patterns after ooulation induction and artificial insemination

In general, oestradiol concentrations increased four- to tenfold after eCG injection (Figs 1 and 5). In the female becoming pregnant after gonadotrophin therapy and artificial insemination, increased progestogen metabolite excretion was sustained throughout the 94 day gestation, although concentrations fluctuated markedly throughout the luteal period (Fig. 5a). In one female that failed to conceive, similar increases in progestogen metabolite concentrations were apparent for 57 days after the gonadotrophin-induced oestradiol surge (Fig. 5b). The individual shown in Fig. $5 \mathrm{c}$ had only two distinct follicles $>2 \mathrm{~mm}$ in diameter and no corpora lutea at the time of laparoscopy and, based on a lack of increased progestogen metabolite excretion, ovulation never occurred in response to hCG. A similar anovulatory profile was observed in this female in the subsequent procedure for induction of ovulation. The steroid excretory 


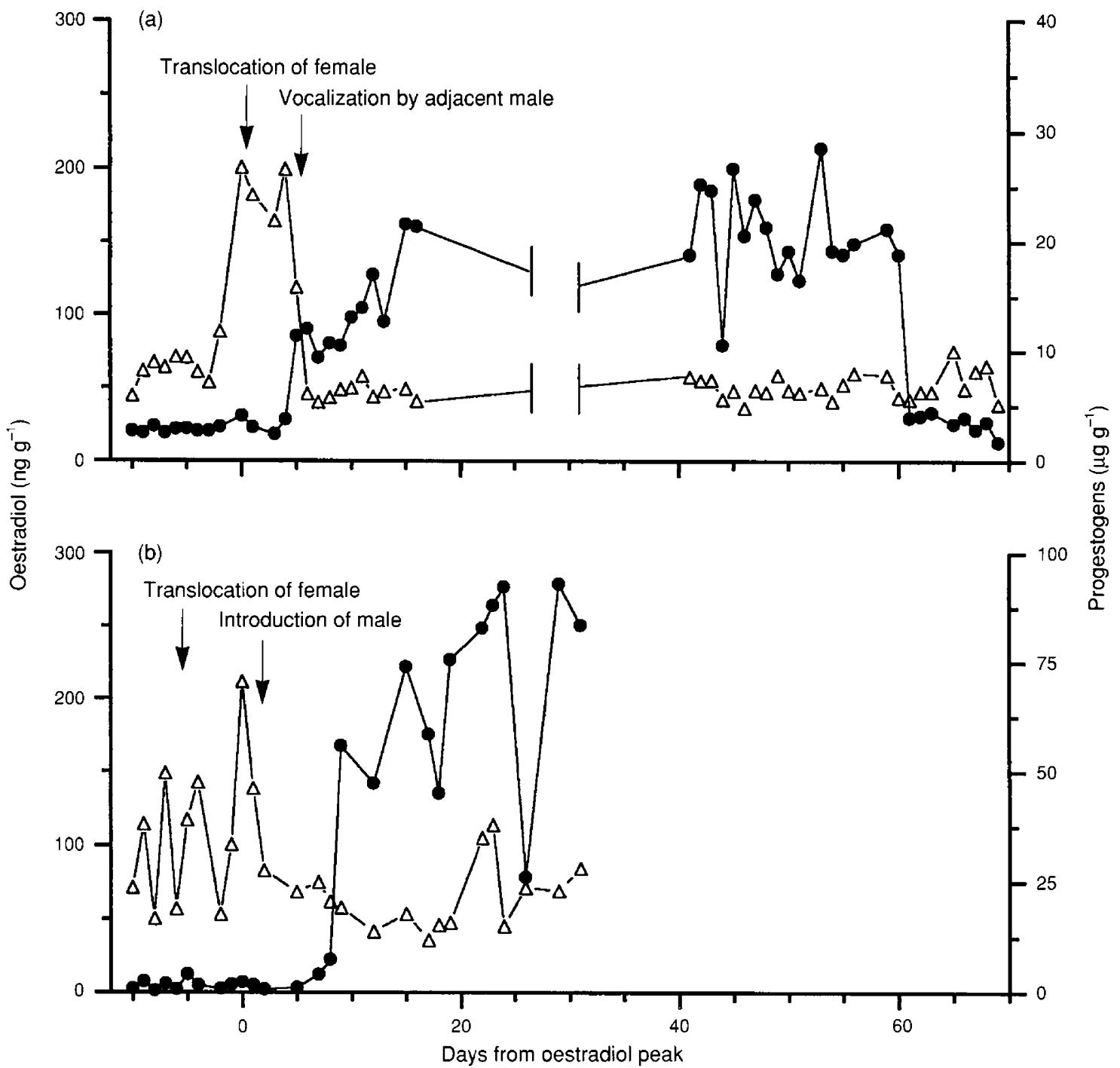

Fig. 4. Individual profiles of faecal oestradiol $(\Delta)$ and progestogen $(\boldsymbol{O})$ metabolite concentrations in cheetah females at (a) the White Oak Conservation Center and (b) Wildlife Safari exhibiting spontaneous ovulation (without mounting or intromission by a male). The female in (a) was relocated to a new enclosure and a male moved into the adjacent enclosure on the same day. Five days later the male showed interest in the female by vocalizing a 'stutter call'. In (b), the female was translocated and a male introduced into her enclosure 1 week later. The male showed interest in the female (calling, approaches to female), but was removed from the enclosure before mounting occurred.

profiles of the remaining individual not conceiving after insemination were similar to that depicted in Fig. 5 b.

\section{Discussion}

Several studies have used faecal oestradiol and progestogen metabolite analyses to examine ovarian activity in cheetahs; however, endocrine assessments were of short duration (<90 days) (Brown et al., 1994; Czekala et al., 1994) or based on only a few animals $(n=5$, Brown et al, 1994; $n=7$, Czekala et al., 1994; $n=2$, Graham et al., 1995). The present study evaluated endocrine patterns for extended periods (up to 24 months) in 26 individuals ( $n=36$ total observations, since ten females were evaluated twice) to examine seasonality, ovulatory mechanisms (spontaneous versus induced) and steroid profiles during pregnancy versus 'pseudopregnancy' (the nonpregnant luteal phase) more comprehensively. This information was then used to evaluate possible causes of poor reproductive performance in captive cheetahs.

In general, the 13.6 day oestrous cycle determined in this study by faecal oestradiol analysis was consistent with the 12-14 day cycle proposed by Eaton and Craig (1973), Bertschinger et al. (1984) and Asa et al. (1992), based on behavioural observations, plasma oestradiol concentrations and vaginal cytology, respectively. Together these data confirm that the cheetah is unique among the 'great' cats in exhibiting a shorter cycle on average than the approximately 20-30 day cycle reported for large felids (lion, Panthera leo, Schmidt et al., 1979; puma, Felis concolor, Bonney et al., 1981; tiger, Panthera 

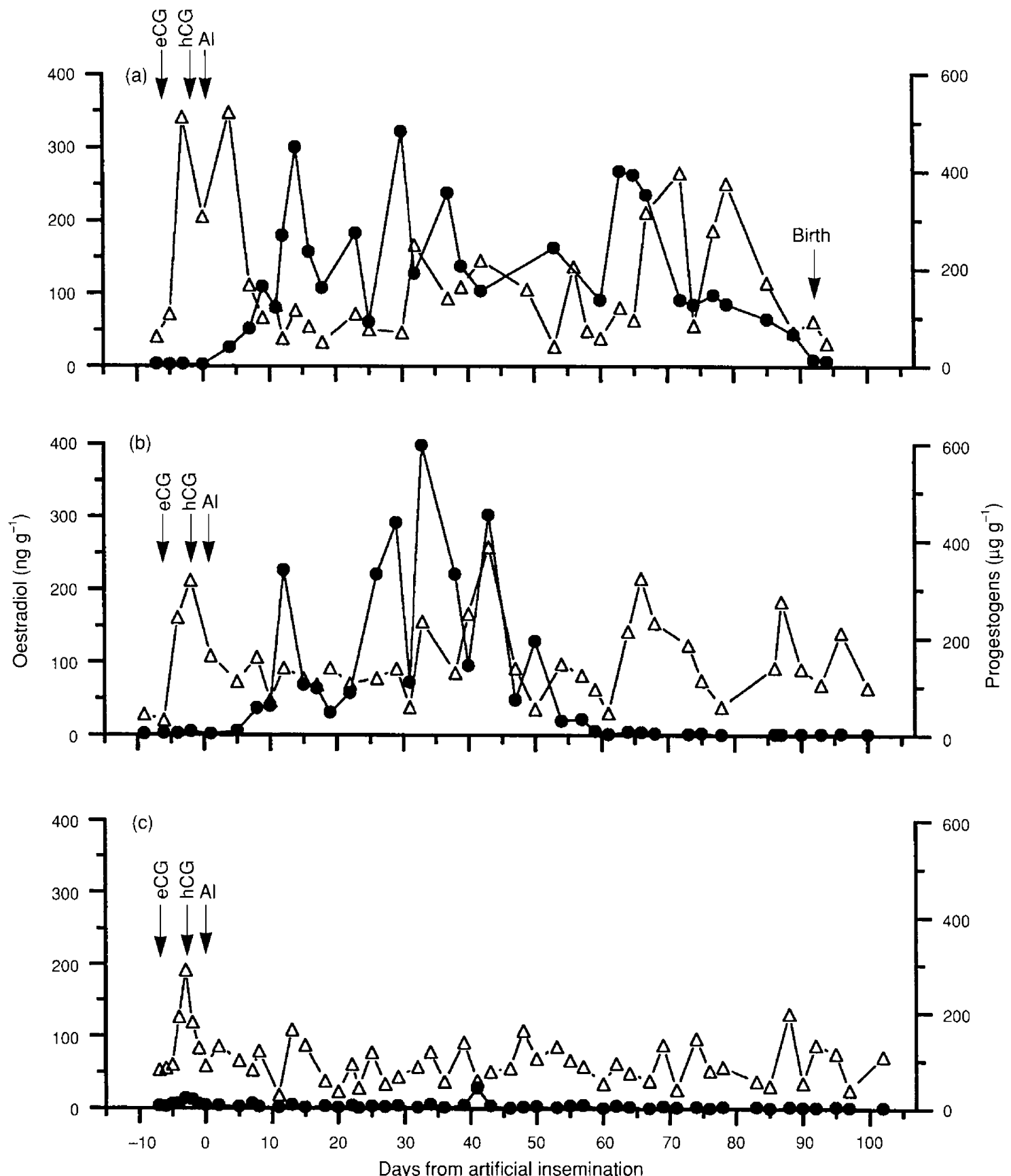

Fig. 5. Individual profiles of faecal oestradiol $(\Delta)$ and progestogen $(-)$ metabolite concentrations in (a) pregnant, (b) nonpregnant and (c) anovulatory female cheetah subjected to ovulation induction and artificial insemination at the Caldwell Zoo. Females were injected i.m. with 200 iu eCG followed $80 \mathrm{~h}$ later by 100 iu hCG and artificial insemination (AI) $46-48 \mathrm{~h}$ after hCG.

tigris, Seal et al., 1985; leopard, Panthera pardus, Schmidt et al., 1988; snow leopard, Panthera uncia, Schmidt et al., 1993; clouded leopard, Neofelis nebulosa, Brown et al., 1995). However even with daily faecal collection, there was considerable variation within and among individuals in oestrous cycle dynamics. Such variability in follicular steroid and behavioural cycle activity is common within species and members of the Felidae (Eaton and Craig, 1973; Kleiman, 1974; Schmidt et al., 1979, 1988, 1993; Bonney et al., 1981; Seal et al., 1985; Yamada and Durrant, 1989; Asa et al., 1993; Brown et al., 1995; Graham et al., 1995) and, in part, may be related to the induced ovulatory characteristics of these species. Compared with spontaneous ovulators, the signalling of oestrus onset and its termination in felids appears to be less finely regulated, making cyclicity more variable.

During pregnancy, progestogen metabolite concentrations increased several hundred-fold above baseline values, peaked about mid-term and then gradually declined until after 
parturition, in a similar way to circulating blood progesterone concentrations in domestic cats (Schmidt et al., 1983). In mated females that failed to conceive, the duration of the nonpregnant luteal phase was about half that of pregnancy. Except for duration of excretion, however, there were no obvious qualitative or quantitative differences in progestogen metabolite profiles between pregnant and nonpregnant cheetahs, which is typical of observations in other felids (domestic cat, Shille and Stabenfeldt, 1979; Wildt et al., 1981; lion, Schmidt et al., 1979; Graham et al., 1995; puma, Bonney et al., 1981; leopard, Schmidt et al., 1988; snow leopard, Schmidt et al., 1993; clouded leopard, Brown et al., 1995). Two of 26 cheetahs in the study population showed evidence of spontaneous (nonmating-induced) ovulations on the basis of increased faecal progestogens after an increase in oestradiol. During the reproductive survey, Wildt et al. (1993) observed no active luteal tissue on the ovaries of nonpregnant or nonlactating females and concluded that cheetahs were induced ovulators. However, distinct luteal scars were found on the ovaries of $12 \%$ of cheetahs that had never produced young, although mating histories were unknown. In other studies, neither Czekala et al. (1994) nor Bertschinger et al. (1984) found evidence of nonmating-induced ovulations, whereas Asa et al. (1992) did quantify a sustained increase in serum concentrations of progesterone in a singleton cheetah. Comparatively, no 'spontaneous' ovulations have been reported in pumas (Bonney et al., 1981), tigers (Seal et al., 1985) or snow leopards (Schmidt et al., 1993), whereas occasional non-mating-induced ovulations have been observed in lions (Schramm et al., 1994) and clouded leopards (Brown et al., 1995; Howard et al., in press) maintained in female groups or as singletons, and in leopard females housed together, but not alone (Schmidt et al., 1988). Taken together, these data demonstrate that, while usually ovulating only after copulation, ovulation in some individual felids can be triggered occasionally by physical or psychosocial stimuli unrelated to mating. Furthermore, although the incidence of spontaneous ovulations may vary among species, for cheetahs it appears to be extremely low.

In the survey of Wildt et al. (1993), females demonstrated minimal, if any, ovarian activity at the time of examination. Although about $67 \%$ of the surveyed population had at least one ovarian follicle $\geq 2 \mathrm{~mm}$ in diameter, only $23 \%$ had ovaries containing follicles considered mature ( $\geq 4 \mathrm{~mm}$ ). Most of these surveyed cheetahs also had low serum oestradiol concentrations, further suggesting they were reproductively inactive (Wildt et al.. 1993). However, this single-point-in-time survey was not designed to evaluate ovarian dynamics. By monitoring longitudinal ovarian steroid excretion, our study found that, over prolonged periods, $75 \%$ of the cheetahs did exhibit some follicular activity. In addition, all females examined for a year or more demonstrated waves of follicular activity for as little as $25 \%$ and up to $80 \%$ of the time. None of this reproductive activity appeared to be seasonally mediated. It now remains to be determined what mediates this discontinuous ovarian cyclicity in cheetahs and how (or if) it affects overall reproductive performance. Several physiological causes were eliminated by the survey of Wildt et al. (1993), which reported no differences in reproductive tract anatomy, pituitary function or gonadal activity between proven and unproven breeders. It also is known that cheetahs exhibit a high degree of genetic monomorphism (O'Brien et al., 1983, 1985) and that loss of heterozygosity reduces overall reproductive fitness in other species (Ralls et al., 1979; Ralls and Ballou, 1983). This reduced genetic diversity probably accounts for the extremely high percentage of morphologically abnormal spermatozoa noted in cheetah electroejaculates (Wildt ef al., 1983, 1987; Wildt, 1994). However, poor ejaculate quality and low genetic diversity are observed in both captive cheetahs and in successfully reproducing free-living cheetahs (O'Brien et al, 1983, 1985; Wildt et al., 1987). Thus, genetic factors alone are not likely to be the major contributors to poor fertility within the captive population. Rather, behavioural problems may be an underlying cause of poor reproductive success in cheetahs (Laurenson et al., 1992; Caro, 1993, 1994). Breeding success is associated with widely varying husbandry practices and no single method has proven successful across institutions (Caro, 1993). Furthermore, the fact that behavioural signs often are difficult to interpret in cheetahs reinforces the need for integrated studies correlating behavioural observations with actual endocrine events. The unexpected finding that animals within the same institution often alternated periods of oestrous cyclicity leads to speculation that reproductive suppression may be occurring among some cheetah females housed together or in close proximity. Although not documented within the Felidae (most of which are solitary), reproductive suppression of subordinates occurs in many social species, including callitrichid primates (Abbott, 1984; Epple and Katz, 1984; French et al., 1984), naked mole rats (Heterocephalus glaber, Faulkes et al., 1990), dwarf mongooses (Helogale parvula, Creel et al., 1992) and African wild dogs (Lycaon pictus, Frame et al., 1979; Fuller et al., 1992). In the wild, female cheetahs tend to travel alone, whereas males live in stable coalitions of 2-3 animals (Laurenson et al., 1992; Caro, 1993, 1994). Keeping males and females together continually in captivity or the absence of male coalitions may be detrimental to promoting natural courtship behaviour in both sexes. This finding warrants further investigation using more controlled experimental procedures.

It is clear that any meaningful evaluation of reproductive status in individual female cheetahs requires long-term evaluation of ovarian activity and emphasizes the power of noninvasive faecal steroid monitoring for assessing reproductive activity. From a practical perspective, faecal steroid analyses will provide critical information needed for making appropriate captive management decisions, especially on how environmental changes or husbandry practices affect reproductive activity. These assays are also an important adjunct tool for assessing ovarian responses to gonadotrophin therapy, allowing for subtle improvements that eventually should permit assisted reproduction to be even more useful for maintaining genetic diversity within smali populations.

The authors thank R. Hoyt and T. Volk of the Phoenix Zoo; C. Marsh and M. Tucker of the Caldwell Zoo; J. Hausjergen, K. Van der Molen and T. Vargas of the Sacramento Zoo; J. Fleming, K. Ziegler and L. Ferguson of the White Oak Conservation Center; T. Peterson, S. Russell and G. Ziegler of the Wildlife Safari; and A. Bellem, H. Tomaso, W. Rowntree and K. Grieg of the Metro Toronto Zoo for logistical support and sample collection. They are also grateful to S. Beekman, K. Terio and A. Moresco for technical assistance. This study was supported by the Friends of the National Zoo, the New Opportunities in Animal Health Sciences Center, the 
Conservation Endowment Fund/Ralston Purina Big Cat Survival Fund of the American Zoo and Aquarium Association and the Scholarly Studies Program of the Smithsonian Institution.

\section{References}

Abbott DH (1984) Behavioural and physiological suppression of fertility in female marmoset monkeys American Journal of Primatology 6 169-186

Asa CS, Junge RE, Bircher SJ, Noble GA, Sarri KJ and Plotka ED (1992) Assessing reproductive cycles and pregnancy in cheetahs (Acinonyx jubatus) by vaginal cytology Zoo Biology 11 139-151

Bertschinger HJ, Meltzer DG, van Dyk A, Coubrough RI, Soley JT and Collett FA (1984) Cheetah life-line Nuclear Active 30 2-7

Bonney RC, Moore HDM and Jones DM (1981) Plasma concentrations of oestradiol-I7 $\beta$ and progesterone, and laparoscopic observations of the ovary in the puma (Felis concolor) during oestrus, pseudopregnancy and pregnancy Journal of Reproduction and Fertility 63 523-531

Brown JL, Wasser SK, Wildt DE and Graham LH (1994) Comparative aspects of steroid hormone metabolism and ovarian activity in felids, measured non-invasively in feces Biology of Reproduction 51 776-786

Brown JL, Wildt DE, Graham LH, Byers AP, Collins L, Barrett S and Howard JG (1995) Natural versus chorionic gonadotropin-induced ovarian responses in the clouded leopard (Neofelis nebulosa) assessed by fecal steroid analysis Biology of Reproduction 53 93-102

Caro TM (1993) Behavioral solutions to breeding cheetahs in captivity: insights from the wild Zoo Biology 12 19-30

Caro TM (1994) Conservation of cheetahs in the wild and captivity. In Cheetahs of the Serengeti Plains: Group Living in an Asocial Species pp 345-367 Ed. GB Schaller. University of Chicago Press, Chicago, Illinois

Creel SR, Creel NM, Wildt DE and Monfort SL (1992) Behavioural and endocrine mechanisms of reproductive suppression in Serengeti dwarf mongooses Animal Behaviour 43 231-245

Czekala NM, Durrant BS, Callison L, Williams M and Millard S (1994) Fecal steroid hormone analysis as an indicator of reproductive function in the cheetah Zoo Biology 13 119-128

Eaton RL and Craig SJ (1973) Captive management and mating behavior of the cheetah. In The World's Cats I: Ecology and Conservation pp 217-254 Ed. RL Eaton. World Wildlife Safari, Winston, Oregon

Epple G and Katz Y (1984) Social influences on estrogen excretion and ovarian cyclicity in saddle-back tamarins American Journal of Primatology 6 215-227

Faulkes CG, Abbott DH and Jarvis JUM (1990) Social suppression of ovarian cyclicity in captive and wild colonies of naked mole rats, Heterocephalus glaber Journal of Reproduction and Fertility 88 559-568

Frame LH, Malcolm JR, Frame GW and van Lawick H (1979) Social organization of African wild dogs (Lycaon pictus) on the Serengeti Plains, Tanzania, 1967-1978. Zeitschrift für Tierpsychology 50 225-249

French JA, Abbott DH, Cheffler G, Robinson J and Goy RW (1984) Cyclic excretion of urinary oestrogens in female tamarins (Sanguinus oedipus) Journal of Reproduction and Fertility 68 177-184

Fuller TK, Kat PW, Bulger JB, Maddock AH, Ginsberg JR, Burrows R, McNutt JW and Mills DR (1992) Population dynamics of African wild dogs. In Wildilife 2001: Populations pp 10-15 Eds DR McCullough and RH Barret. Elsevier Applied Science, New York

Graham LH, Goodrowe KL, Raeside JI and Liptrap RM (1995) Non-invasive monitoring of ovarian function in several felid species by measurement of fecal estradiol-17 $\beta$ and progestins Zoo Biology 14 223-237

Guggisburg CAW (1975) Cheetah, hunting leopard, Acinonyx jubatus. In Wild Cats of the World pp 260-289 Ed CAW Guggisburg. Taplinger, New York

Howard JG, Donohgue AM, Barone MA, Goodrowe KL, Blumer ES, Snodgrass K, Starnes D, Tucker M, Bush M and Wildt DE (1992) Successful induction of ovarian activity and laparoscopic intrauterine artificial insemination in the cheetah (Acinonyx jubatus) Journal of Zoo and Wildlife Medicine 23 288-300

Howard JG, Byers AP, Brown JL، Barrett SJ, Evans MZ, Schwartz RJ and Wildt DE Successful ovulation induction and laparoscopic intrauterine artificial insemination in the clouded leopard (Neofelis nebulosa) Zoo Biology (in press)
Kleiman DG (1974) The estrus cycle in the tiger (Panthera tigris). In The World's Cats, Vol. 2. Biology, Behavior and Management of Reproduction pp 60-75 Ed. RL Eaton. Feline Research Group, Seattle, WA

Laurenson MK, Caro T and Borner M (1992) Female cheetah reproduction National Geographic Research and Exploration 8 64-75

Marker L and O'Brien SJ (1989) Captive breeding of the cheetah (Acinonyx jubatus) in North American zoos (1871-1986) Zoo Biology 8 3-16

Marker-Kraus L and Grisham J (1993) Captive breeding of cheetahs in North American zoos (1987-1991) Zoo Biology 12 5-18

O'Brien SJ, Wildt DE, Goldman CR, Merril CR and Bush M (1983) The cheetah is depauperate in genetic variation Science 221 459-462

O'Brien SJ, Roelke ME, Marker L, Newman A, Winkler CA, Meltzer D, Colly L, Bush M, Evermann JR and Wildt DE (1985) Genetic basis for species vulnerability in the cheetah Science 227 1428-1434

Ralls K and Ballou J (1983) Extinction: lessons from zoos. In Genetics and Conservation: A Reference for Managing Wild Animal and Plant Populations pp 35-56 Eds CM Schonewald-Cox, SM Chambers, B MacBryde and L Thomas. Benjamin-Cummings, Menlo Park, CA

Ralls K, Brugger K and Ballou J (1979) Inbreeding and juvenile mortality in small populations of ungulates Science 206 1101-1103

Risler L, Wasser SK and Sackett GP (1987) Measurement of excreted steroids in Macaca nemestrina American Journal of Primatology 12 91-100

Schmidt AM, Nadal LA, Schmidt MJ and Beamer NB (1979) Serum concentrations of oestradiol and progesterone during the normal oestrous cycle and early pregnancy in the lion (Panthera leo) Journal of Reproduction and Fertility $\mathbf{5 7}$ 267-272

Schmidt AM, Hess DL, Schmidt MJ, Smith RC and Lewis CR (1988) Serum concentrations of oestradiol and progesterone, and sexual behavior during the normal oestrous cycle in the leopard (Panthera pardus) Journal of Reproduction and Fertility 82 43-49

Schmidt AM, Hess DL, Schmidt MJ and Lewis CR (1993) Serum concentrations of oestradiol and progesterone and frequency of sexual behaviour during the normal oestrous cycle in the snow leopard (Panthera uncia) Journal of Reproduction and Fertility 98 91-95

Schmidt PM, Chakraborty PK and Wildt DE (1983) Ovarian activity, circulating hormones and sexual behavior in the cat. l. Relationships during pregnancy, parturition, lactation and the postpartum estrus Biology of Reproduction $\mathbf{2 8}$ 657-671

Schramm RD, Briggs MB and Reeves JJ (1994) Spontaneous and induced ovulation in the lion (Panthera leo) Zoo Biology 13 301-307

Seal US, Plotka ED, Smith JD, Wright FH, Reindl N, Taylor RS and Seal MF (1985) Immunoreactive luteinizing hormone, estradiol, progesterone, testosterone and androstenedione levels during the breeding season and anestrus in Siberian tigers Biology of Reproduction 32 361-368

Shille VM and Stabenfeldt GH (1979) Luteal function in the domestic cat during pseudopregnancy and after treatment with prostaglandin $F_{24 t}$ Biology of Reproduction 21 1217-1223

Wildt DE (1994) Endangered species spermatozoa: diversity, research and conservation. In Function of Somatic Cells in the Testes pp 1-24 Ed. A Bartke. Springer Verlag, New York

Wildt DE, Chan SYW, Seager SWJ and Chakraborty PK (1981) Ovarian activity, circulating hormones and sexual behavior in the cat. I. Relationships during the coitus-induced luteal phase and the estrous period without mating Biology of Reproduction 25 15-28

Wildt DE, Bush M, Howard JG, O'Brien SJ, Meltzer D, van Dyk, Ebedes $\mathrm{H}$ and Brand DJ (1983) Unique seminal quality in the South African cheetah and a comparative evaluation in the domestic cat Biology of Reproduction 29 1019-1025

Wildt DE, O'Brien SJ, Howard JG, Caro TM, Roelke ME, Brown JL and Bush M (1987) Similarity in ejaculate-endocrine characteristics in captive versus free-ranging cheetahs of two subspecies Biology of Reproduction 36 351-360

Wildt DE, Brown JL, Bush M, Barone MA, Cooper KA, Grisham J and Howard JG (1993) Reproductive status of cheetahs (Acinonyx jubatus) in North American zoos: the benefits of physiological surveys for strategic planning Zoo Biology $1245-80$

Yamada JK and Durrant BS (1989) Reproductive parameters of clouded leopards (Neofelis nebulosa) Zoo Biology 8 223-231 